\title{
Performance of one-view breast tomosynthesis as a stand-alone breast cancer screening modality: results from the Malmö Breast Tomosynthesis Screening Trial, a population-based study
}

\author{
Kristina Lång ${ }^{1}$ - Ingvar Andersson ${ }^{1}$ - Aldana Rosso ${ }^{2}$ - Anders Tingberg ${ }^{3}$. \\ Pontus Timberg ${ }^{3} \cdot$ Sophia Zackrisson ${ }^{1}$
}

Received: 23 January 2015 /Revised: 20 March 2015 / Accepted: 14 April 2015 /Published online: 1 May 2015

(C) The Author(s) 2015. This article is published with open access at Springerlink.com

\begin{abstract}
Objective To assess the performance of one-view digital breast tomosynthesis (DBT) in breast cancer screening. Methods The Malmö Breast Tomosynthesis Screening Trial is a prospective population-based one-arm study with a planned inclusion of 15000 participants; a random sample of women aged 40-74 years eligible for the screening programme. This is an explorative analysis of the first half of the study population $(\mathrm{n}=7500)$. Participants underwent one-view DBT and two-view digital mammography (DM), with independent double reading and scoring. Primary outcome measures were detection rate, recall rate and positive predictive value (PPV). McNemar's test with $95 \%$ confidence intervals was used. Results Breast cancer was found in sixty-eight women. Of these, 46 cases were detected by both modalities, 21 by DBT alone and one by DM alone. The detection rate for one-view DBT was 8.9/1000 screens (95\% CI 6.9 to 11.3 ) and 6.3/1000 screens (4.6 to 8.3) for two-view DM ( $p<$ $0.0001)$. The recall rate after arbitration was $3.8 \%$ (3.3 to 4.2) for DBT and $2.6 \%$ (2.3 to 3.0) for DM ( $<<0.0001)$. The PPV was $24 \%$ for both DBT and DM.
\end{abstract}

Kristina Lång

kristina.lang@med.lu.se

1 Department of Diagnostic Radiology, Translational Medicine Malmö, Lund University, Inga Marie Nilssons gata 49, SE20502 Malmö, Sweden

2 Epidemiology and Register Centre South, Skåne University Hospital, Klinikgatan 22, SE-221 85 Lund, Sweden

3 Department of Medical Radiation Physics, Translational Medicine Malmö, Lund University, Inga Marie Nilssons gata 49, SE20502 Malmö, Sweden
Conclusion Our results suggest that one-view DBT might be feasible as a stand-alone screening modality.

Key Points

- One-view DBT as a stand-alone breast cancer screening modality has not been investigated.

- One-view DBT increased the cancer detection rate significantly.

- The recall rate increased significantly but was still low.

- Breast cancer screening with one-view DBT as a stand-alone modality seems feasible.

Keywords Mammography · Screening · Diagnostic Imaging $\cdot$ Breast Cancer $\cdot$ Women' Health

\section{Introduction}

Population-based breast cancer screening with digital mammography (DM) has the potential to reduce breast cancer mortality [1]. Still, 15-30 \% of all cancers may be missed, mainly due to the anatomic noise of the breast, i.e., normal breast tissue overlapping and obscuring the lesion of interest $[2,3]$. This applies especially to dense breasts [4]. In recent years, digital breast tomosynthesis (DBT) has been developed as a three-dimensional mammographic technique with the aim to reduce the adverse effect of overlapping tissue [5, 6]. Typically, a DM screen is obtained in two projections. The DBT images can be acquired in either one or two projections, referred to as one-view or two-view DBT. Several clinical studies involving enriched populations have shown that the accuracy of DBT is superior to DM, demonstrated both when using one and two views [7-10]. Furthermore, data from two large population-based screening trials showed that the use of twoview DBT in combination with two-view DM substantially improved cancer detection compared to two-view DM 
screening, albeit with conflicting results on recall rates [11, 12]. In the United States, a decrease in recall rate was demonstrated when using two-view DBT in combination with twoview DM in large retrospective multicentre screening studies $[13,14]$. The use of one-view DBT or DBT alone in screening has not hitherto been investigated.

The Malmö Breast Tomosynthesis Screening Trial (MBTST) is the first trial designed to assess the efficacy of one-view DBT versus two-view DM, and a combination of one-view DBT and one-view DM versus two-view DM, in breast cancer screening. In this explorative study comprising the first half of the study population, we present our first results on screening performance measures from the MBTST.

\section{Methods}

\section{Study design}

The MBTST is a prospective one-arm single-institution study investigating the use of one-view DBT (mediolateral oblique (MLO)) alone versus two-view DM (craniocaudal (CC) and MLO), and a combination of one-view DBT (MLO) and oneview $\mathrm{DM}(\mathrm{CC})$ in a population-based screening programme in the city of Malmö, Sweden (www.clinicalTrials.gov; NCT01091545). The study was approved by the Regional Ethical Review Board at Lund University (Dnr 2009/770) and the local Radiation Safety Board at Skåne University Hospital in Malmö. Participating women gave written informed consent. All participants were offered one-view DBT (MLO) and two-view DM (Mammomat Inspiration, Siemens AG, Erlangen, Germany) in a single session upon arrival at the screening unit. Women who declined to participate in the study had the DM examination only. This is an explorative analysis of the first half of the study population with a complete set of DBT and DM screens, i.e., 7500 participants. Outcome measures for this report were breast cancer detection rate, recall rate, and positive predictive value.

\section{Population}

All women aged 40-74 years are included in the Swedish breast cancer screening programme, in accordance with recommendations from the National Board of Health and Welfare [15]. Women aged 40-55 years are invited every 18 months and women aged 56-74 years every 24 months. A random sample of women eligible for the ordinary screening programme in the city of Malmö were invited to participate in the study by a letter added to the screening invitation. Women were chosen from the population-based screening registry with the primary aim of obtaining a representative sample of the population in terms of age distribution. Upon arrival at the screening unit, the women received further written and oral information about the study, and if they agreed to participate, gave written consent. The exclusion criteria were pregnancy and women not speaking Swedish or English. The first half of the study population was included from January 2010 to December 2012.

\section{Image acquisition}

Two-view DM images followed by one-view DBT images were acquired at a single occasion. DBT images were acquired using the same beam quality and anode/filter combination (W/Rh) as DM. The automatic exposure control was set to yield an average glandular dose of $1.2 \mathrm{mGy}$ for DM and $1.6 \mathrm{mGy}$ for DBT (for a standard breast model with an equivalent breast thickness of $53 \mathrm{~mm}$ (simulated by a $45 \mathrm{~mm}$ PMMA phantom) measured according to EUREF draft QC protocol for breast tomosynthesis systems version 1.5 (www. EUREF.org) including a forward scatter factor (1.06) of the compression paddle) $[16,17]$. Hence, the absorbed dose in a one-view DBT examination is approximately $70 \%$ of the absorbed dose in a two-view DM. Each DBT view consisted of 25 projection images acquired over an angular range of 50 degrees. The images were reconstructed into $1 \mathrm{~mm}$ slices using a generalised filtered back-projection routine [18]. The DBT examination was performed with reduced compression of the breast compared to the previously acquired DM image set, with a goal of a $50 \%$ reduction [19]. This was achieved in most cases (about $90 \%$ ), but sometimes more pressure was needed to keep especially large breasts in a proper position for the MLO projection.

\section{Reading procedures}

Six readers (five readers with more than 10 years of experience in breast radiology and one reader with less than 10 years of experience, mean 26 years, range 8 to 41 years) participated in the trial. They had undergone individual training in interpretation of DBT images and had participated in previous reading studies $[9,10]$. Readers had also been exposed to DBT as part of clinical problem-solving during a 2year period prior to the start of the current study.

The reading included blinded double reading and scoring in two independent reading arms: Reading arm DBT and Reading arm DM (Fig. 1). The readers were randomly assigned to the two reading arms. The findings for DBT and DM were classified using a 5-point scale: (1) normal, (2) benign findings, (3) non-specific finding with low probability of malignancy (4) findings suspicious of malignancy, and (5) findings highly suspicious of malignancy. The scale is similar to the American College of Radiology's Breast Imaging Reporting and Data System (BI-RADS) [20]. The scores were registered in the Radiology Information System (RIS, Sectra, Linköping, Sweden). If one or both of the screening 


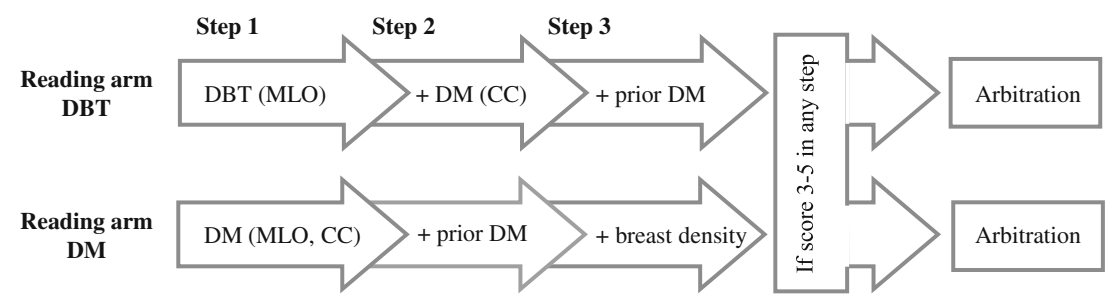

Fig. 1 Reading procedure flowchart. Blinded double reading and scoring in independent reading arms. Each step was scored before proceeding to the next step. A positive score (3-5) in any step qualified the case for arbitration. Prior DM was a two-view DM. DBT=digital breast tomosynthesis, $\mathrm{DM}=$ digital mammography, $\mathrm{MLO}=$ mediolateral oblique view, $\mathrm{CC}=$ craniocaudal view modalities was given a score of 3 or higher by one of the two readers, it was referred for arbitration, where at least two readers re-evaluated the images and decided whether to recall the woman for further work-up, irrespective of the score on the other modality. In the event of a woman reporting symptoms during the screen examination, e.g., palpable lump or nipple secretion, the woman could be recalled in spite of negative imaging findings. Breast density was judged by one reader in step 3 in Reading arm DM, and classified according to BI-RADS: (1) fatty, (2) scattered fibroglandular densities, (3) heterogeneously dense, and (4) dense [20].

\section{Workup}

Women recalled for assessment had their workup done at the breast clinic by the same group of radiologists who were involved in the reading of the DBT and DM screening images. The assessment usually included additional DM images and an ultrasound examination and, in the event of a lesion, also a needle biopsy. Outcomes were assessed by needle biopsy, surgery or at least one-year follow-up. All cancer cases were verified through record linkage with the South Swedish Cancer Registry.

\section{Statistical analysis}

The MBTST was designed to include approximately 15,000 women in order to get an $80 \%$ power, with a two-sided significant threshold of $5 \%$, to demonstrate an increased sensitivity from about $63 \%$ when using DM to $88 \%$ when using DBT, in a population with a cancer prevalence of 6 per 1000 women. Also, the sample size calculation took into account an assumed increase in false positive fraction of $20 \%$ with an equivalent specificity for DM and DBT of $4 \%$. The calculations were based on Receiver Operating Characteristic statistical analysis [21]. In order to evaluate the progress of the clinical trial and the feasibility of our assumptions in the sample size calculations, we performed this explorative analysis with screening performance measures of the first half of the study population. The inclusion of participants with a complete set of DBT and DM examinations gives paired binary data for all participants. McNemar's test was used to analyse the paired data of DBT and DM screens for differences in detection and recall rates with $95 \%$ CIs. Differences in characteristics between cancers detected solely by DBT and all DM-detected cancers were tested using chi-2 test and Fisher's exact test, if the sample size was small. Analyses were done with Stata software (version 13).

\section{Results}

Of 10,547 women invited, 7500 participated in the study (71.1\%) (Fig. 2). The mean age of the participants was 56 years (range 40-76). The number of participants screened for the first time was estimated to be 1563 women, i.e., $20 \%$. The breast density distribution in the study population according to BI-RADS was fatty: $20 \%$; scattered densities: $38 \%$; heterogeneously dense: $34 \%$, and dense: $8 \%$.

Sixty-seven women were detected with breast cancer in Reading arm DBT and 47 in Reading arm DM, respectively. Of these, 46 cancer cases were detected in both reading arms. Hence, 21 cancer cases were detected by DBT alone and one cancer by DM alone. The detection rate for Reading arm DBT and Reading arm DM was 8.9 per 1000 screens (95\% CI 6.9 to 11.3 ) and 6.3 per 1000 screens (4.6 to 8.3 ), respectively. The increase in cancer detection when using DBT relative to DM was $43 \%$ (95\% CI 21 to $68 ; p<0.0001)$. All cancers in Reading arm DBT were detected in the first reading step, i.e. by DBT alone. Hence, there were no additional cancers detected by the combination of one-view DBT and one-view DM, i.e. reading step 2 in the DBT reading arm.

The number of recalled women in Reading arm DBT was 282 and in Reading arm DM 197, respectively. The recall rate for Reading arm DBT and Reading arm DM was 3.8 \% (95\% CI 3.3 to 4.2 ) and $2.6 \%$ (2.3 to 3.0), respectively. The increase in recall rate when using DBT relative to DM was $43 \%(95 \%$ CI 26 to $62 ; p<0.0001)$. The positive predictive value was $24 \%(67 / 282)$ for DBT as well as for DM (47/197).

The recall rates and number of cancers per reading arm, related to the positive score before arbitration for each reading step, are illustrated in Table 1.

All cancers were verified microscopically on surgical specimens, except for one case verified with core biopsy (refused 
Fig. 2 Study population flow chart. DBT $=$ digital breast tomosynthesis, $\mathrm{DM}=$ digital mammography

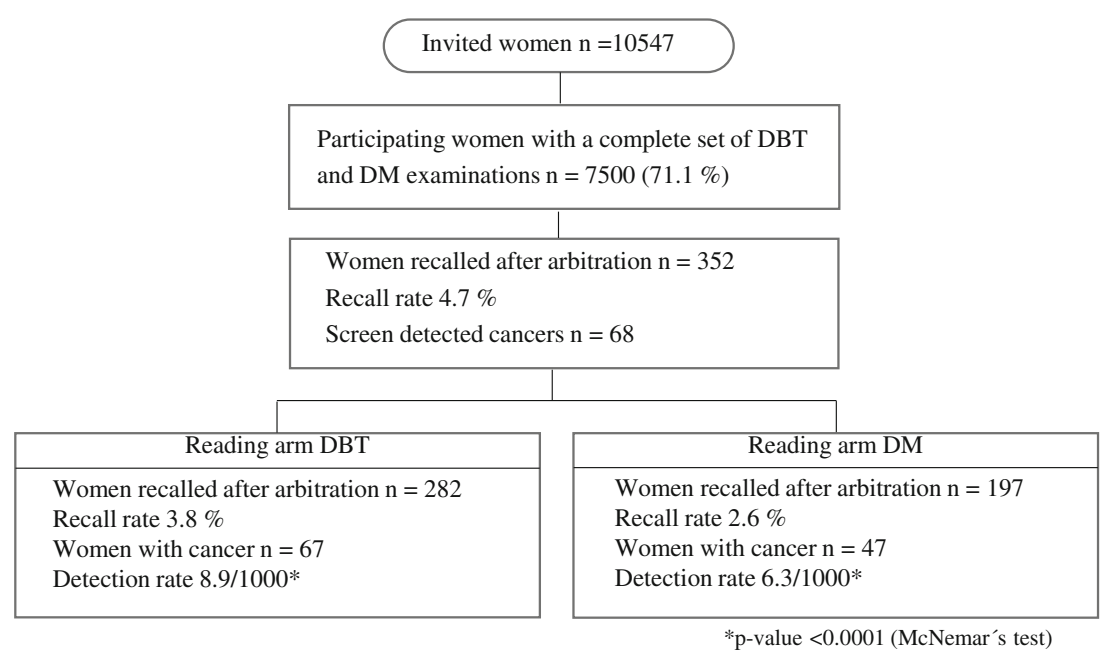

surgery). The characteristics of the cancers are summarised in Table 2.

Seventeen out of 21 DBT alone detected cancers were invasive, ten were grade 2 or 3 and two had lymph node metastases. There were no statistically significant differences in cancer characteristics between cases detected by DBT alone and cancers detected by DM in regard to proportions of invasive and in situ cancer, cancer type, histological grade, lymph node status, breast density, and tumour size. Still, the cancers detected by DBT alone tended to be more of histological grade 1, lymph node negative, and of smaller size than DM-detected cancers. Also, these women were slightly younger. The cases detected by DBT alone were distributed across all BI-RADS density categories. A typical cancer detected by DBT alone is illustrated in Fig. 3.

\section{Discussion}

The main findings of this explorative analysis suggest that one-view DBT may be feasible as a stand-alone technique for breast cancer screening. Compared with the current gold

Table 1 Results of reading procedure by reading arm and reading step. Reading arm DBT: (reading step 1) one-view DBT alone, (reading step 2) the addition of a DM CC-view, (reading step 3) comparison with prior standard (DM), DBT was superior in terms of detection rate and equal in terms of PPV. The DM CC-view did not contribute to cancer detection but did help in ruling out cancer in a few cases with stable, benign lesions, as did previous DM examinations.

The DBT cancer detection rate may seem remarkably high. However, the current intervention should be regarded as prevalence DBT screening. If a new screening technique, which is superior to DM in terms of cancer detection, is applied, an effect similar to the one observed in the randomised mammography screening trials will be seen, i.e., a higher detection rate in the intervention arm compared to the control arm [22]. In addition, in the current study, approximately $20 \%$ of the population was invited to mammography screening for the first time, either because of turning 40 or being a newly arrived immigrant. As a consequence, it is reasonable to expect a lower detection rate if subsequent screening rounds were performed.

In the present study there is not enough data for a statistical comparison of the biological characteristics of the DBT and DM detected cancers. Even though the rate of detected DCIS was similar in the two reading arms, the cancers detected by

two-view DM, if available. Reading arm DM: (reading step 1) two-view $\mathrm{DM}$, (reading step 2) comparison with prior DM. DBT=digital breast tomosynthesis, DM=digital mammography

\begin{tabular}{|c|c|c|c|c|c|c|}
\hline \multirow[b]{2}{*}{ Positive score before arbitration } & \multicolumn{3}{|c|}{ Reading arm DBT } & \multicolumn{3}{|c|}{ Reading arm DM } \\
\hline & Recalls (n) & Recall rate & Cancers (n) & Recalls (n) & Recall rate & Cancers (n) \\
\hline Reading step 1 & 243 & $3.2 \%$ & 67 & 166 & $2.2 \%$ & 47 \\
\hline Reading step 2 & 10 & $0.1 \%$ & 0 & 2 & 0.02 & 0 \\
\hline Reading step 3 & 4 & $0.05 \%$ & 0 & $\mathrm{n} / \mathrm{a}$ & $\mathrm{n} / \mathrm{a}$ & $\mathrm{n} / \mathrm{a}$ \\
\hline Symptoms, no imaging finding & 25 & $0.3 \%$ & 0 & 29 & 0.04 & 0 \\
\hline Total & 282 & $3.8 \% *$ & $67 \dagger$ & 197 & $2.6 \% *$ & $47 \dagger$ \\
\hline
\end{tabular}

*p-value $<0.0001$ (McNemar's test)

$\dagger$ p-value $<0.0001$ (McNemar's test) 
Table 2 Summary of cancer characteristics. DBT=digital breast tomosynthesis, DM=digital mammography

\begin{tabular}{|c|c|c|c|c|}
\hline & Detected DBT total & Detected DBT alone* & Detected DM total* & Detected DM alone \\
\hline Total & 67 & 21 & 47 & 1 \\
\hline Total invasive cancers & $58(87)$ & $17(81)$ & $41(87)$ & - \\
\hline Total in situ cancers & $9(13)$ & $4(19)$ & $6(13)$ & $1(100)$ \\
\hline \multicolumn{5}{|l|}{ Cancer type } \\
\hline IDC & $37(55)$ & $10(48)$ & $27(57)$ & - \\
\hline DCIS + microfoci of IDC & $3(4)$ & $1(5)$ & $2(4)$ & - \\
\hline DCIS & $7(10)$ & $3(14)$ & $5(11)$ & $1(100)$ \\
\hline ILC & $9(13)$ & $3(14)$ & $6(13)$ & - \\
\hline Tub & $9(13)$ & $3(14)$ & $6(13)$ & - \\
\hline LCIS & $2(3)$ & $1(5)$ & $1(2)$ & - \\
\hline \multicolumn{5}{|l|}{ Histological grade } \\
\hline Grade 1 & $24(36)$ & $10(48)$ & $14(30)$ & - \\
\hline Grade 2 & $23(34)$ & $5(24)$ & $19(40)$ & $1(100)$ \\
\hline Grade 3 & $18(23)$ & $5(24)$ & $13(28)$ & - \\
\hline $\mathrm{n} / \mathrm{a}$ & $2(3)$ & $1(5)$ & $1(2)$ & - \\
\hline \multicolumn{5}{|l|}{ Lymph node status } \\
\hline Positive & $14(21)$ & $2(10)$ & $12(26)$ & - \\
\hline Negative & $50(74)$ & $18(86)$ & $32(68)$ & $1(100)$ \\
\hline $\mathrm{n} / \mathrm{a}$ & $3(4)$ & $1(5)$ & $3(6)$ & - \\
\hline Mean size (range) & $16 \mathrm{~mm}(5-60)$ & $13 \mathrm{~mm}(6-25)$ & $17 \mathrm{~mm}(5-60)$ & $8 \mathrm{~mm}$ \\
\hline Mean age (range) & 60 yrs (40-74) & 58 yrs (43-71) & 61 yrs $(40-74)$ & $65 \mathrm{yrs}$ \\
\hline \multicolumn{5}{|l|}{ Breast density } \\
\hline Fatty & $7(10)$ & $3(14)$ & $4(9)$ & - \\
\hline Scattered & $17(25)$ & $5(24)$ & $12(26)$ & - \\
\hline Heterogeneous & $33(49)$ & $10(48)$ & $24(51)$ & $1(100)$ \\
\hline Dense & $10(15)$ & $3(14)$ & $7(15)$ & - \\
\hline \multicolumn{5}{|l|}{ Radiographic appearance } \\
\hline Spiculated mass & $51(76)$ & $16(76)$ & $35(74)$ & - \\
\hline Circumscribed mass & $8(12)$ & $3(14)$ & $5(11)$ & - \\
\hline Architectural distortion & $1(1)$ & $1(5)$ & $0(0)$ & - \\
\hline Microcalcifications & $7(10)$ & $1(5)$ & $7(15)$ & $1(100)$ \\
\hline
\end{tabular}

Data are $\mathrm{n}(\%)$ or range when stated

$\mathrm{IDC}=$ invasive ductal carcinoma, $\mathrm{DCIS}=$ ductal carcinoma in situ, $\mathrm{ILC}=$ invasive lobular carcinoma, $\mathrm{Tub}=$ invasive tubular carcinoma,

LCIS $=$ lobular carcinoma in situ

$\left.{ }^{*}\right)$ There were no statistically significant differences between the characteristics of the cancers detected by DBT alone and DM total

DBT alone tended to be of lower grade, smaller size and lymph node negative, compared with DM-detected cancers. Also, these women were slightly younger at diagnosis. It is not clear whether this represents earlier diagnosis and/or overdiagnosis from DBT screening, since the present study was not designed to address these issues. This question will be further analysed when the entire study is completed. Our impression in the present study, as well as from a previous study, is that DBT is particularly sensitive to the detection of small spiculated lesions [23]. Such lesions tend to be either lowgrade cancers or benign radial scars, while small high-grade cancers tend to be less specific in appearance, i.e., non- specific densities with diffuse borders or well circumscribed with benign appearance [24]. Furthermore, the cancers detected by DBT alone were found in both dense and fatty breasts, which mean that the increased cancer detection rate with DBT is not solely due to the reduction of the negative effect of superimposed tissue in dense breasts but also to better lesion conspicuity in more fatty breasts. These results are in accordance with findings in the two earlier prospective screening trials [11, 12]. Accordingly, DBT will also enhance benign lesions and sometimes islands of normal breast parenchyma. This probably contributed to the significant increase in recall rate with DBT compared to DM. Even if the recall rate was 


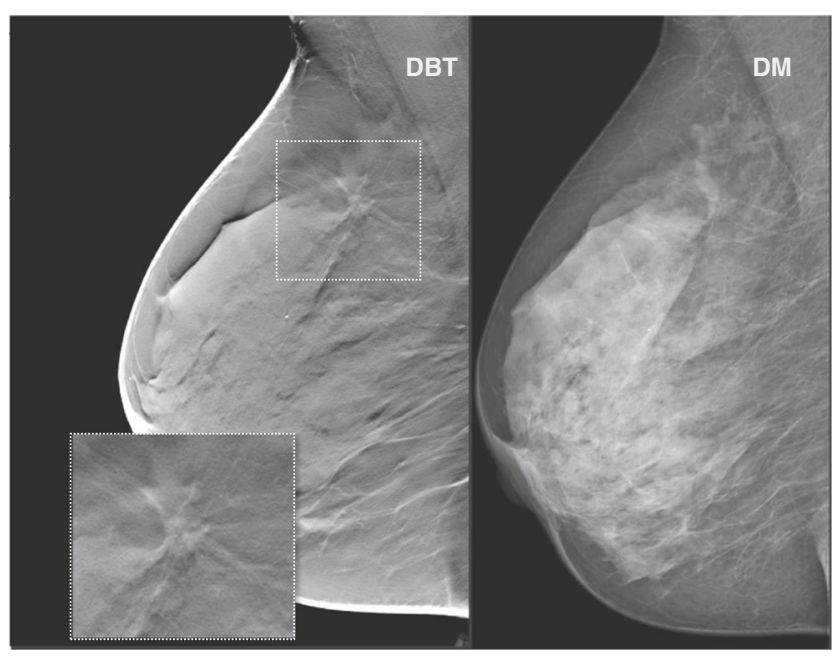

Fig. 3 Cancer detected by digital breast tomosynthesis alone. A sixtysix-year-old asymptomatic woman recalled for findings suspected only on digital breast tomosynthesis. A $15-\mathrm{mm}$ invasive ductal carcinoma, histological grade 1 and lymph node negative, was diagnosed at histological examination

higher with DBT, it was still low and in agreement with the recommendation from the European Guidelines for Quality Assurance in Breast Cancer Screening and Diagnostic Services [25]. Several studies have reported a reduced false positive rate with DBT. However, the baseline recall rate was much higher in these studies compared with MBTST [11, 13, 14]. Furthermore, the increased recall rate observed in this study was higher than assumed in the sample size calculation. It is reasonable to expect that the increase in recall rate will be somewhat lower in the second half of the study population in line with increased experience of the readers. Further analysis on recall rates will be presented after the completion of the MBTST.

\section{Implications for practice}

In general, a mass screening technique should be fast, with a high sensitivity and specificity, reasonably inexpensive, and, in the case of an X-ray examination, at a low radiation dose. Accordingly, in the design of the MBTST we chose one-view DBT in the MLO-projection. This projection has been shown to be the most effective in mammography screening [26]. We estimate that the reading time for one-view DBT in screening is about doubled compared to DM. We did not register the reading time in our study, since the reading and scoring procedures were specific to the trial and would not reflect the true time consumption in a normal screening workflow. In a previous study from our group, we found that the reading time for one-view DBT (MLO) was roughly $30 \mathrm{~s}$ (in an enriched population of clinical and screening cases) [27]. This is about one third of the reported reading time for the so-called combination mode - two-view DBT in combination with two-view DM - used in the Oslo Tomosynthesis Screening Trial [12]. In our study, the radiation dose for one-view DBT examination was lower than an ordinary two-view DM screen, which is satisfactory from a mass screening perspective. Especially considering that the use of a combination mode, evaluated in the other screening trials, gives additional radiation dose compared to a single modality approach $[11,12]$. Still, the use of a synthetic mammogram derived from the DBT volume can provide means to sustain a low radiation dose even with the use of a combination mode.

The reduced compression force used in this study was much appreciated by the women, according to our examining technologists. However we did not collect any qualitative data to verify this statement. The high cancer detection rate in the current study supports previous results showing that reduced compression does not significantly compromise image quality [19]. We believe this is an important advantage of DBT.

Breast cancer mammography screening has been considered cost-effective [28]. The cost-effectiveness of DBT compared to $\mathrm{DM}$ in screening for breast cancer has yet to be evaluated, and is important for future decisions on the introduction of DBT in screening. So far, it is reasonable to assume that DBT will be more expensive, but this has to be related to the benefit of increased cancer detection, and probably earlier detection.

The ultimate measure of screening efficacy is the reduction of breast cancer mortality. Our study was not designed to assess differences in breast cancer mortality, for that purpose a very large randomised screening trial would be needed. An alternative could be to analyse the relative reduction of interval cancers as a surrogate end-point for screening efficacy. An estimation of interval cancer rates will be included in future reports from the MBTST, after the inclusion and follow-up of the whole study population.

\section{Limitations}

A limitation of this study is that it was performed on an urban Swedish population, and might, therefore, not be representative for other populations. In addition, the examinations were performed using one particular brand of tomosynthesis equipment that differs from other commercially available systems. Also, the readers involved had participated in previous clinical studies on the accuracy of DBT, and might, therefore, be biased in favour of DBT. As a consequence, this explorative analysis will not introduce additional bias to the readers. Furthermore, the limitation of an explorative analysis on half of the study population is that it does not have an $80 \%$ power in the statistical analyses.

\section{Conclusion}

We found a significant increase in cancer detection rate when using one-view DBT as a stand-alone screening modality compared to two-view DM. The recall rate increased 
significantly but was still low. If one-view DBT is to be used in mass screening, advantages such as reduced compression force and lower radiation dose can be gained. Our results suggest that one-view DBT might be feasible as a standalone breast cancer screening modality.

Acknowledgments The radiologists participating in the screen reading were Annicka Lindahl, Marianne Löfgren, Cecilia Wattsgård, and Barbara Ziemiecka, and the nurses responsible for performing the screening examinations were Ulrica Pettersson and Maria Seserin.

The scientific guarantor of this publication is Sophia Zackrisson. The authors of this manuscript declare relationships with the following companies: Siemens AG (Erlangen, Germany) sponsored the study by providing the breast tomosynthesis equipment. KL, IA, SZ, PT, and AT have received speakers' fees and travel grants from Siemens. This study has received funding by Swedish Research Council, Swedish Cancer Society, Skane county council's research and development foundation, Lund University Medical Faculty, Unilabs, Sweden, and Siemens. One of the authors has significant statistical expertise (AR). Institutional Review Board approval was obtained. Written informed consent was obtained from all subjects (patients) in this study. Methodology: prospective, diagnostic or prognostic study, performed at one institution.

Open Access This article is distributed under the terms of the Creative Commons Attribution-NonCommercial 4.0 International License (http:// creativecommons.org/licenses/by-nc/4.0/), which permits any noncommercial use, distribution, and reproduction in any medium, provided you give appropriate credit to the original author(s) and the source, provide a link to the Creative Commons license, and indicate if changes were made.

\section{References}

1. Independent UK Panel on Breast Cancer Screening (2012) The benefits and harms of breast cancer screening: an independent review. Lancet 380:1778-1786

2. Laming D, Warren R (2000) Improving the detection of cancer in the screening of mammograms. J Med Screen 7:24-30

3. Bochud FO, Valley JF, Verdun FR et al (1999) Estimation of the noisy component of anatomical backgrounds. Med Phys 26:13651370

4. Carney PA, Miglioretti DL, Yankaskas BC et al (2003) Individual and combined effects of age, breast density, and hormone replacement therapy use on the accuracy of screening mammography. Ann Intern Med 138:168-175

5. Baker JA, Lo JY (2011) Breast tomosynthesis: state-of-the-art and review of the literature. Acad Radiol 18:1298-310

6. Niklason LT, Christian BT, Niklason LE et al (1997) Digital tomosynthesis in breast imaging. Radiology 205:399-406

7. Houssami N, Skaane P (2013) Overview of the evidence on digital breast tomosynthesis in breast cancer detection. Breast 22:101-108

8. Lei J, Yang P, Zhang L et al (2013) Diagnostic accuracy of digital breast tomosynthesis versus digital mammography for benign and malignant lesions in breasts: a meta-analysis. Eur Radiol 24:595-602

9. Andersson I, Ikeda DM, Zackrisson S et al (2008) Breast tomosynthesis and digital mammography: a comparison of breast cancer visibility and BIRADS classification in a population of cancers with subtle mammographic findings. Eur Radiol 18:2817-2825

10. Svahn TM, Chakraborty DP, Ikeda D et al (2012) Breast tomosynthesis and digital mammography: a comparison of diagnostic accuracy. Br J Radiol 85:e1074-82
11. Ciatto S, Houssami N, Bernardi D et al (2013) Integration of 3D digital mammography with tomosynthesis for population breastcancer screening (STORM): a prospective comparison study. Lancet Oncol 14:583-589

12. Skaane P, Bandos AI, Gullien R et al (2013) Comparison of digital mammography alone and digital mammography plus tomosynthesis in a population-based screening program. Radiology 267:47-56

13. Haas BM, Kalra V, Geisel J et al (2013) Comparison of tomosynthesis plus digital mammography and digital mammography alone for breast cancer screening. Radiology 269: 694-700

14. Friedewald SM, Rafferty EA, Rose SL et al (2014) Breast cancer screening using tomosynthesis in combination with digital mammography. JAMA 311:2499-507

15. The National Board of Health and Welfare. (2007) [The National Guidelines for breast-, colorectal and prostate cancer]. Stockholm, Sweden. Available via http://www.socialstyrelsen.se/ nationellariktlinjerforbrostprostatatjocktarmsochandtarm scancervard. Accessed 9 Sep 2014

16. Van Engen R, Bouwman R, Dance D, Heid P, Lazzari B, Marshall N, Schopphoven S, Strudley C, Thjissen M, Young K BH (2013) Protocol for the quality control of the physical and technical aspects of digital breast tomosynthesis system. EUREF, European Guidelines for Quality Assurance in Breast Cancer Screening and Diagnosis

17. Hemdal B (2011) Forward-scattered radiation from the compression paddle should be considered in glandular dose estimations. Radiat Prot Dosim 147:196-201

18. Mertelmeier T (2006) Optimizing filtered backprojection reconstruction for a breast tomosynthesis prototype device. Proc SPIE. doi:10.1117/12.651380

19. Förnvik D, Andersson I, Svahn T, et al. The effect of reduced breast compression in breast tomosynthesis: human observer study using clinical cases. Radiat Prot Dosimetry 139:118-23

20. D’Orsi CJ, Mendelson EB, Morris EASEA (2013) ACR BIRADS $^{\circledR}$ Atlas, Breast Imaging Reporting and Data System. American College of Radiology, Reston, VA

21. Sullivan M (2003) The statistical evaluation of medical tests for classification and prediction, 2nd edn. Oxford University Press Inc, New York, pp 230-233

22. Tabar L, Fagerberg CJ, Gad A et al (1985) Reduction in mortality from breast cancer after mass screening with mammography. Randomised trial from the Breast Cancer Screening Working Group of the Swedish National Board of Health and Welfare. Lancet 1:829-832

23. Förnvik D, Zackrisson S, Ljungberg O et al (2010) Breast tomosynthesis: Accuracy of tumor measurement compared with digital mammography and ultrasonography. Acta Radiol 51:240 247

24. Evans AJ, Pinder SE, James JJ et al (2006) Is mammographic spiculation an independent, good prognostic factor in screeningdetected invasive breast cancer? AJR Am J Roentgenol 187: $1377-1380$

25. Perry N, Broeders M, de Wolf C et al (2008) European guidelines for quality assurance in breast cancer screening and diagnosis. Fourth edition-summary document. Ann Oncol 19:614-622

26. Lundgren B, Helleberg A (1982) Single oblique-view mammography for periodic screening for breast cancer in women. $\mathrm{J}$ Natl Cancer Inst 68:351-355

27. Dustler M, Förnvik D, Timberg P, Tingberg A (2013) A Study of the Feasibility of using slabbing to reduce Tomosynthesis Review Time. Proc SPIE. doi:10.1117/12.2006987

28. Stout NK, Rosenberg MA, Trentham-Dietz A et al (2006) Retrospective cost-effectiveness analysis of screening mammography. J Natl Cancer Inst 98:774-82 